\title{
Correlação entre intensidade da dor e incapacidade com as lesões intra-articulares em pacientes com síndrome do impacto femoroacetabular
}

\section{Correlation between Intensity of Pain and Disability Due to Intra-articular Lesions in Patients with Femoroacetabular Impingement Syndrome}

\author{
Giancarlo Cavalli Polesello ${ }^{1}$ Nayra Deise Anjos Rabelo ${ }^{2(}$ João Tomás Fernandes Castilho Garcia ${ }^{3(0)}$ \\ Walter Ricioli Junior ${ }^{10}$ Marco Rudelli ${ }^{1 \odot}$ Marcelo Cavalheiro de Queiroz ${ }^{1 \odot}$
}

${ }^{1}$ Grupo de Quadril, Departamento de Ortopedia e Traumatologia, Faculdade de Ciências Médicas, Santa Casa de Misericórdia de São Paulo (FCMSCSP), São Paulo, SP, Brasil

2 Núcleo de Apoio à Pesquisa em Análise do Movimento (NAPAM), Universidade Nove de Julho, São Paulo, SP, Brasil

${ }^{3}$ Residência Médica, Departamento de Ortopedia e Traumatologia, Faculdade de Ciências Médicas, Santa Casa de Misericórdia de São Paulo, (FCMSCSP), São Paulo, SP, Brasil,

\begin{abstract}
Endereço para correspondência Giancarlo Cavalli Polesello, M.D, Ph. D., Adjunct professor and head of the hip group, Departamento de Ortopedia e Traumatologia da Santa Casa de São Paulo, "Pavilhão Fernandinho Simonsen”, Sala do Grupo do Quadril ( $2^{\circ}$ andar), Rua Dr. Cesário Motta Junior, 112, Bairro Vila Buarque, São Paulo, SP, 01221. 020, Brasil (e-mail: giancarlopolesello@hotmail.com).
\end{abstract}

Rev Bras Ortop 2022;57(5):836-842.

\section{Resumo \\ Palavras-chave \\ - impacto femoroacetabular \\ - artroscopia \\ - dor \\ - quadril}

Objetivo Correlacionar alterações radiográficas e lesões de estruturas intra-articulares do acetábulo com a intensidade da dor e a incapacidade de pacientes com diagnóstico de síndrome do impacto femoroacetabular.

Métodos Realiou-se uma análise retrospectiva de dados pré-operatórios de 182 pacientes (190 quadris). Foram avaliadas variáveis clínicas como idade, sexo e prática de atividade física, e variáveis radiográficas, como ângulo de Wiberg e o ângulo alfa. Por meio do vídeo intraoperatório, foi avaliada a extensão das lesões condrais e labiais considerando-se o método clock-face, o grau de comprometimento articular pela classificação de Outerbridge, e a presença de lesão em onda. As variáveis foram analisadas por meio de regressão linear, tendo como variáveis dependentes a intensidade da dor, avaliada pela Escala Visual Analógica (EVA), e a incapacidade funcional, mensurada pelo Harris Hip Score modificado (HHSm).

Resultados A média de idade dos pacientes foi de $38,5 \pm 9,6$ anos, a da intensidade da dor, $7,8 \pm 1,6$, e a do HHSm, 56,3 $\pm 12,7$. No total, $61 \%$ da amostra apresentava Outerbridge III ou IV, e 12,6\% apresentava lesão em onda. Observou-se correlação do sexo masculino $(r=0,497)$ com menor intensidade da dor, e correlação da idade

\footnotetext{
Trabalho realizado no Grupo de Quadril do Departamento de Ortopedia e Traumatologia da Faculdade de Ciências Médicas Santa Casa de Misericórdia de São Paulo (FCMSCSP), São Paulo, SP, Brasil.
}

recebido

22 de Julho de 2020

aceito

03 de Novembro de 2020

Publicado on-line

de Janeiro 21, 2022
DOI https://doi.org/

10.1055/s-0041-1729573. ISSN 0102-3616.
(C) 2022. Sociedade Brasileira de Ortopedia e Traumatologia. All rights reserved.

This is an open access article published by Thieme under the terms of the Creative Commons Attribution-NonDerivative-NonCommercial-License, permitting copying and reproduction so long as the original work is given appropriate credit. Contents may not be used for commercial purposes, or adapted, remixed, transformed or built upon. (https://creativecommons.org/ licenses/by-nc-nd/4.0/)

Thieme Revinter Publicações Ltda., Rua do Matoso 170, Rio de Janeiro, RJ, CEP 20270-135, Brazil 


\author{
Abstract \\ Keywords \\ - femoroacetabular \\ impingement \\ - arthroscopy \\ - pain \\ - hip
}

$(r=-0,27)$, do sexo masculino $(r=8,419)$ e da realização de atividade física com maior escore funcional no HHSm $(r=4,729)$.

Conclusão Não houve correlação dos parâmetros radiográficos e artroscópicos deste estudo com a intensidade da dor e a incapacidade dos pacientes. O sexo masculino está relacionado com menor intensidade da dor, e maior capacidade funcional está relacionada com o sexo masculino, menor idade, e a prática de atividade física.

Nível de Evidência IV.

Objective To correlate radiographic alterations and lesions in intra-articular structures of the acetabulum with the intensity of pain and disability of patients diagnosed with femoroacetabular impingement syndrome.

Methods A retrospective analysis of the preoperative data of 182 patients (190 hips) was performed. Clinical variables such as age, gender, the practice of physical activity, and radiographic variables, such as the Wiberg and alpha angles, were evaluated. Through an intraoperative video, the extent of the chondral and labial lesions was evaluated considering the clock-face method, the degree of joint involvement by the Outerbridge classification, and the presence of wave lesions. The variables were analyzed by linear regression, with the intensity of the pain assessed by the Visual Analog Scale (VAS), and functional disability measured by the Modified Harris Hip Score (mHHS).

Results The mean age of the patients was of $38.5 \pm 9.6$ years, the mean intensity of the pain was of $7.8 \pm 1.6$, and the mean mHHS score was of $56.3 \pm 12.7$. In total, $61 \%$ of the sample were classified as Outerbridge III or IV, and $12.6 \%$ had wave lesions. There was a correlation between the male gender $(r=0.497)$ and lower intensity of the pain, and a correlation of age $(r=-0.27)$, the male gender $(r=8.419)$ and physical activity with higher functional scores on the mHHS $(r=4.729)$.

Conclusion There was no correlation of the radiographic and arthroscopic parameters of the present study and the intensity of pain and the disability of the patients. The male gender is related to lower intensity of pain, and higher functional ability is related to the male gender, lower age, and the practice of physical activity.

Level of Evidence IV.

\section{Introdução}

A síndrome do impacto femoroacetabular (SIFA) é uma condição clínica que consiste no impacto precoce entre o fêmur proximal e o acetábulo, ${ }^{1}$ e é uma causa comum de dor no quadril e incapacidade física em indivíduos adultos jovens. ${ }^{2,3}$ É descrita como um distúrbio clínico do quadril relacionado ao movimento, em que o indivíduo apresenta uma tríade de sinais e sintomas clínicos, que envolve dor, limitação da amplitude de movimento do quadril e incapacidade funcional, atrelada aos achados de imagem característicos de alterações morfológicas na junção colo-cabeça do fêmur, ou impacto do tipo cam, no rebordo acetabular, ou impacto do tipo pincer, ou ambos, conhecido como impacto do tipo misto. ${ }^{4}$

Sabe-se que uma das queixas mais comuns relacionadas à SIFA é a dor do quadril, sobretudo na região inguinal, mas esses indivíduos também sofrem déficit funcional, dificuldade para a realização das atividades de vida diária e esportivas, limitação do arco de movimento, e piora da qualidade de vida. ${ }^{3,4}$ Além disso, de acordo com a patogênese dessa condição, o contato precoce entre as estruturas ósseas já mencionadas, durante os movimentos como flexão, adução e rotação interna, pode levar a impactação e consequente lesão de estruturas articulares, como o lábio e a cartilagem. Isso resulta em danos articulares progressivos, ${ }^{5}$ relacionados a doenças degenerativas como a artrose do quadril e dor. ${ }^{6}$ Desta forma, acredita-se que o diagnóstico precoce e a precisa indicação do tratamento têm papel determinante na melhora do quadro.

Apesar de plausível, a associação entre a morfologia alterada ou o dano dos tecidos moles articulares ${ }^{1}$ e a manifestação dos sintomas e incapacidade funcional dos indivíduos com SIFA ainda permanece incerta. A prevalência de patologias intra-articulares dos tecidos moles que se acredita estarem associadas à SIFA foi relatada em uma revisão recente, ${ }^{7}$ e os dados indicam que lesões labrais foram observadas em $62 \%$ dos indivíduos sintomáticos e em $54 \%$ dos assintomáticos. Por outro lado, defeitos de cartilagem foram relatados em $64 \%$ dos sintomáticos, em comparação com $12 \%$ nos assintomáticos. Já edema ósseo subcondral e lesões no 
ligamento redondo foram mais prevalentes em pessoas com sintomas, e cistos paralabrais e cistos de impacto foram igualmente prevalentes tanto em sintomáticos quanto em assintomáticos.

A maior prevalência desses achados em indivíduos com SIFA pode reforçar a ideia de que a dor e a incapacidade evidenciada nesses casos têm relação direta com os achados de imagem. Contudo, os poucos estudos ${ }^{8,9}$ que testaram a relação da morfologia óssea e do dano tecidual articular com a intensidade da dor nos sujeitos com SIFA não evidenciaram uma correlação importante.

Isso mostra que ainda não está clara a interação entre a dor e comprometimento funcional com as lesões estruturais encontradas nessa doença, e que, apesar da melhora clínica evidenciada após a cirurgia artroscópica, ${ }^{10}$ o entendimento dos fatores associados à dor e à incapacidade desses pacientes ainda permanece incerto. Sendo assim, os objetivos deste estudo foram: analisar a correlação entre as alterações morfológicas estudadas e o grau de lesão das estruturas intra-articulares com a dor e a função de pacientes que estavam aguardando procedimento cirúrgico para SIFA; e identificar os fatores associados à dor e à função desses indivíduos.

\section{Métodos}

O trabalho consiste no estudo retrospectivo dos dados préoperatórios dos pacientes com diagnóstico de SIFA com indicação para a cirurgia artroscópica do quadril. Estes são dados pré-operatórios extraídos de uma série consecutiva de pacientes pertencente ao banco de dados de procedimentos artroscópicos realizados por um único cirurgião especialista em cirurgia do quadril, com vasta experiência em artroscopia do quadril, em um período de oito anos, cujos dados pósoperatórios foram usados em outro estudo do mesmo grupo de autores deste artigo, com cadastro no comitê de ética (CAAE: 49250215.4.0000.5479).

\section{Critérios de Inclusão e Exclusão}

Um segundo cirurgião, com cerca de cinco anos de experiência em cirurgia ortopédica e em processo de aperfeiçoamento em cirurgia do quadril, analisou retrospectivamente os prontuários, e revisou os vídeos das artroscopias dos pacientes com diagnóstico de SIFA e lesão labral que foram submetidos a procedimento artroscópico do quadril. Foram excluídos pacientes com alterações displásicas congênitas do quadril, como displasia do desenvolvimento do quadril, pela relação já definida de mau prognóstico, ${ }^{11}$ pacientes com osteoartrose do quadril que levasse à diminuição do espaço articular menor do que $2 \mathrm{~mm}$, pacientes com lesão traumática do lábio que já tenham sido submetidos a outros procedimentos cirúrgicos do quadril, e pacientes com preenchimento incompleto do prontuário.

\section{Variáveis Mensuradas}

Foram consideradas como variáveis dependentes a intensidade da dor pré-operatória, analisada por meio da Escala Visual Analógica (EVA), ${ }^{12}$ e a capacidade funcional pré- operatória, analisada por meio do Harris Hip Score modificado (HHSm) por Byrd, questionário traduzido e adaptado para a língua portuguesa. ${ }^{13}$

As variáveis independentes analisadas foram: idade, sexo, lateralidade, prática de atividade física, ângulo alfa, ângulo de Wiberg, extensão da lesão labral e condral pelo método clock-face, lesão em onda, e o grau de lesão condral pela classificação de Outerbridge.

Foi considerado praticante de atividade física o indivíduo que realizava 150 minutos de atividade moderada por semana no mínimo, conforme as recomendações do American College of Sports Medicine. ${ }^{14}$

\section{Análise Radiográfica}

Realizou-se uma análise radiográfica dos quadris dos pacientes incluídos para a medida do ângulo de Wiberg (ângulo centro-borda, $C B)^{15}$ e do ângulo alfa em radiografias em incidência anteroposterior (AP). ${ }^{6}$ As medidas foram realizadas por meio de radiografias digitais com o uso do programa IMPAX Orthopaedic Tools (AGFA HealthCare, Mortsel, Bélgica), versão 1.0 .323 .

O ângulo de Wiberg, ou CB, é utilizado para a avaliação da cobertura da cabeça proporcionada pelo teto ósseo acetabular. Ele foi obtido ao se traçar uma linha que cruza os centros das cabeças femorais, uma perpendicular à essa linha, passando também pelo centro da cabeça femoral, e uma outra linha no rebordo lateral do acetábulo. ${ }^{12}$

O ângulo alfa é uma medida proposta por Nötzli et al. ${ }^{16}$ que avalia a concavidade da junção fêmur-cabeça. Ele foi obtido por meio da intersecção de duas linhas, traçadas ao longo eixo do colo femoral, e uma linha traçada do centro da cabeça femoral até o ponto de maior perda da esfericidade da cabeça.

\section{Análise dos Danos Condrais e Labrais}

Por meio da análise do vídeo do intraoperatório artroscópico, o mesmo cirurgião ortopedista (com cinco anos de experiência) que realizou a inclusão dos pacientes no estudo avaliou a extensão das lesões labrais e a extensão das lesões condrais, que foram medidas e localizadas pelo método clockface, sendo a região superior do acetábulo adotada como $12 \mathrm{~h}$, e a extensão, calculada com base na distância em "horas" entre as extremidades lesionais. ${ }^{17}$

As classificação de Outerbridge das lesões condrais também foi determinada por meio da análise do vídeo do procedimento artroscópico, ${ }^{18}$ e elas foram classificadas nos graus de 1 a 4, com um espectro de lesões que variavam desde edema da cartilagem até erosão da cartilagem subcondral. ${ }^{19}$

Em relação aos danos condrais, durante o procedimento foi realizada a "palpação" da cartilagem articular com sonda artroscópica para localizar alterações sugestivas de delaminação da cartilagem articular com perda da fixação da cartilagem ao osso subcondral, que caracterizam a lesão em onda (-Figura 1). ${ }^{20,21}$

\section{Análise Estatística}

A normalidade das variáveis quantitativas de desfecho principal foi testada pelo teste de Kolmogorov-Smirnov (KS). O 
teste de igualdade de duas proporções foi aplicado para caracterizar a distribuição da frequência relativa (percentuais) das variáveis qualitativas.

O coeficiente de correlação de Spearman foi calculado para se avaliar a associação das pontuações no HHSm e na EVA com as covariáveis quantitativas, e, para analisar as covariáveis qualitativas em relação às mesmas pontuações, foi realizado o teste de Mann-Whitney. Por fim, foi realizada uma análise multivariada de regressão linear seguindo o modelo stepwise para verificar quais variáveis produzem efeito nas pontuações no HHSm e na EVA. Todas as análises estatísticas foram realizadas usando-se o programa Statistical Package for the Social Sciences (SPSS, IBM Corp., Armonk, NY, US), versão 20.0, com significância definida como valores de $p<0,05$, e intervalos com $95 \%$ de confiança estatística.

\section{Resultados}

Foram analisados um total de 182 pacientes (190 quadris), com média de idade de 38,5 (desvio padrão [DP]: 9,8) anos, sendo que, destes, 58,4\% eram homens, com média de dor de 7,8 (DP: 1,6) pontos na EVA e pontuação de 56,3 (DP: 12,7) no HHSm. Ao todo, 61\% da amostra apresentava graus III e IV de lesão condral pela classificacão de Outerbridge, e 12,6\% apresentava lesão em onda. Outros dados clínicos e demográficos e a distribuição de frequência estão descritos nas - Tabelas $\mathbf{1}$ e $\mathbf{2}$, respectivamente.

Na análise de correlação com as variáveis quantitativas, foi identificada correlação fraca e negativa entre a pontuação no HHSm e a idade, com r de -0,237. Não foi observada correlação significante entre a intensidade da dor e qualquer variável quantitativa neste estudo.

De acordo com o teste de Mann-Whitney, foi evidenciado efeito estatístico do sexo e da prática de atividade física nos resultados tanto do HHSm quanto da EVA, sendo que pacientes do sexo masculino e aqueles que praticavam atividade física apresentaram maior pontuação funcional e menor intensidade de dor do que pacientes do sexo feminino e indivíduos sedentários $(p=0,011)$. Houve uma tendência de os indivíduos com lesão em onda apresentarem menor pontuação funcional $(p=0,058)$; ainda assim, não houve diferença estatística para essa variável (-Tabela 3). Ao analisar o a classificação de Outebridge com relação ao

Tabela 1 Média e desvio padrão dos dados de caracterização da amostra

\begin{tabular}{|l|l|}
\hline & Média (desvio padrão) \\
\hline Idade & $38,5(9,8)$ \\
\hline Harris Hip Score modificado & $56,3(12,7)$ \\
\hline Escala Visual Analógica & $7,8(1,6)$ \\
\hline Ângulo de Wiberg & $39,1(6,7)$ \\
\hline Ângulo alfa & $57,4(9,3)$ \\
\hline Soma do clock-face labral & $2,53(0,79)$ \\
\hline Soma do clock-face condral & $2,7(0,87)$ \\
\hline
\end{tabular}

Tabela 2 Distribuição das frequências absoluta e relativa dos dados qualitativos

\begin{tabular}{|l|l|l|}
\hline \multicolumn{2}{|l|}{ Dados qualitativos } & N (\%) \\
\hline \multirow{2}{*}{ Prática de atividade física } & Não & $60(31,6)$ \\
\cline { 2 - 3 } & Sim & $130(68,4)$ \\
\hline \multirow{2}{*}{ Lado } & Direito & $112(58,9)$ \\
\cline { 2 - 3 } & Esquerdo & $78(41,1)$ \\
\hline \multirow{3}{*}{ Sexo } & Feminino & $79(41,6)$ \\
\cline { 2 - 3 } & Masculino & $111(58,4)$ \\
\hline \multirow{3}{*}{ Lesão em onda } & Não & $166(87,4)$ \\
\cline { 2 - 3 } & Sim & $24(12,6)$ \\
\hline \multirow{2}{*}{ Grau na Classificação } & I & $40(21,1)$ \\
\cline { 2 - 3 } & II & $34(17,9)$ \\
\cline { 2 - 3 } & III & $55(28,9)$ \\
\cline { 2 - 3 } & IV & $61(32,1)$ \\
\hline
\end{tabular}

HHSm e à EVA, pelo teste de Kruskal-Wallis, não foi identificada interação estatística entre essas variáveis.

Por fim, na análise multivariada de regressão linear, avaliando os modelos pelo método stepwise para a EVA, apenas a variável sexo $(r=-0,497)$ foi significante, ou seja, ser homem é fator relacionado a menor intensidade de dor. Já na análise com relação ao HHSm, 3 variáveis emergiram no modelo como significantes: idade $(r=-0,270)$, sendo que, quanto maior a idade, menor a pontuação funcional; sexo $(r=8,419)$, sendo que, ser homem está relacionado com pontuação funcional mais alta; e atividade física $(\mathrm{r}=4,729)$, que indica uma pontuação funcional maior.

\section{Discussão}

As alterações morfológicas encontradas na SIFA, sobretudo a deformidade do tipo cam, parecem apresentar relação com o comprometimento dos tecidos intra-articulares do quadril durante o impacto ocorrido entre as estruturas ao longo da execução dos movimentos. ${ }^{6}$ É plausível que essas lesões sejam sugestivas da dor e incapacidade funcional evidenciadas nos indivíduos com SIFA. Porém, os achados deste estudo não permitiram encontrar correlação dos parâmetros avaliados, sejam eles anatômicos ou de lesões intra-articulares, com a incapacidade dos pacientes, não sendo identificada correlação entre os achados radiográficos ou a magnitude das lesões labrais e condrais acetabulares observadas no intra-operatório e a intensidade da dor ou incapacidade funcional medida pelo HHSm.

A relação entre as alterações estruturais observadas nos indivíduos com SIFA e as manifestações clínicas dessa síndrome intrigam clínicos e pesquisadores há alguns anos. Frank et al. ${ }^{22}$ avaliaram a presença de alterações estruturais compatíveis com impacto femoroacetabular (IFA) e lesão labral em exames de imagem de pacientes assintomáticos, e identificaram que $37 \%$ deles apresentavam alterações morfológicas do tipo cam, e $67 \%$, do tipo pincer. Heerey 
Tabela 3 Análise multivariada de regressão linear para as variáveis dependentes: dor e Harris Hip Score modificado

\begin{tabular}{|c|c|c|}
\hline \multirow[t]{2}{*}{ Escala Visual Analógica } & \multicolumn{2}{|l|}{ Stepwise } \\
\hline & Coeficiente beta & Valor de $p$ \\
\hline Constante & 8,051 & $<0,001$ \\
\hline Idade & 0,017 & 0,196 \\
\hline Ângulo de Wiberg & 0,021 & 0,265 \\
\hline Ângulo alfa & 0,023 & 0,12 \\
\hline Clock-face labral & $-0,104$ & 0,594 \\
\hline Clock-face condral & 0,139 & 0,435 \\
\hline Sexo masculino* & $-0,537$ & 0,03 \\
\hline Lado direito & $-0,012$ & 0,961 \\
\hline Prática de atividade física & $-0,372$ & 0,178 \\
\hline Lesão em onda & 0,362 & 0,367 \\
\hline Classificação de Outebrigde & $-0,083$ & 0,542 \\
\hline Análise de variância & \multicolumn{2}{|l|}{0,03} \\
\hline $\begin{array}{l}\text { Coeficiente de } \\
\text { determinação }\left(R^{2}\right)\end{array}$ & \multicolumn{2}{|l|}{$2,00 \%$} \\
\hline \multirow{2}{*}{$\begin{array}{l}\text { Harris Hip Score } \\
\text { modificado }\end{array}$} & \multicolumn{2}{|l|}{ Stepwise } \\
\hline & Coeficiente beta & Valor de $p$ \\
\hline Constante & 58,074 & $<0,001$ \\
\hline Idade* & $-0,241$ & 0,006 \\
\hline Ângulo de Wiberg & $-0,224$ & 0,081 \\
\hline Ângulo alfa & $-0,07$ & 0,492 \\
\hline Clock-face labral & 2,209 & 0,105 \\
\hline Clock-face condral & $-0,424$ & 0,731 \\
\hline Sexo masculino* & 7,569 & $<0,001$ \\
\hline Lado direito & $-1,553$ & 0,373 \\
\hline Prática de atividade física* & 4,471 & 0,019 \\
\hline Lesão em onda & $-2,396$ & 0,389 \\
\hline Classificação dde Outebrigde & $-1,188$ & 0,208 \\
\hline Análise de variância & \multicolumn{2}{|l|}{$<0,001$} \\
\hline $\begin{array}{l}\text { Coeficiente de } \\
\text { determinação }\left(R^{2}\right)\end{array}$ & \multicolumn{2}{|l|}{$17,30 \%$} \\
\hline
\end{tabular}

Nota: *Variável com significância estatística.

et al., em uma revisão sistemática com metanálise, constatou por moderada evidência a prevalência de 54\% de lesão labral e de $12 \%$ de lesão condral em indivíduos assintomáticos.

Yamauchi et al. ${ }^{8}$ correlacionaram a sintomatologia e a incapacidade de pacientes com achados radiográficos sugestivos de SIFA, mas não evidenciaram associação entre as alterações nos exames de imagem e a intensidade da dor ou os testes clínicos provocativos. E em estudo conduzido por Grace et al., ${ }^{9}$ observou-se apenas fraca associação entre alterações condrais na cabeça do fêmur e a intensidade da dor de indivíduos com SIFA, ao passo que a extensão das lesões labrais e o dano da cartilagem articular acetabular não se correlacionavam com a sintomatologia dos pacientes.
Sabe-se que a abordagem cirúrgica amplamente indicada para o tratamento da SIFA é a artroscopia, um procedimento que objetiva minimizar as alterações da morfologia óssea e os danos nas estruturas moles, partindo do pressuposto de que estas alterações têm relação direta com o quadro de dor e o comprometimento funcional dos pacientes. ${ }^{23,24}$ No entanto, apesar de este procedimento demonstrar bons resultados, com boa satisfação dos pacientes no pós-operatório, ${ }^{25,26}$ permanece a dúvida quanto aos mecanismos de dor envolvidos com na SIFA, e permanece a grande dificuldade diagnóstica, dado que a SIFA é um transtorno dinâmico multifatorial, e inúmeros fatores contribuintes dela não são avaliados por exames estáticos.

Acreditamos que nossos achados refletem a natureza multidimensional da dor, sobretudo em indivíduos com dor crônica do quadril, nos quais, além de aspectos físicos, fatores psicológicos, como ansiedade, depressão, crenças disfuncionais sobre seu quadro de dor e saúde mental, podem contribuir para a magnitude e cronificação dos sintomas e da incapacidade. ${ }^{27,28}$

Além disso, a ausência de correlação entre as lesões estruturais avaliadas e a dor contesta a visão linear e tradicional dos modelos de compreensão da dor com base apenas no dano tecidual, fato destacado por Jacobs et al., ${ }^{28}$ que, ao avaliarem variáveis relacionadas aos sintomas pré-operatórios de indivíduos com SIFA, identificaram que pontuações de saúde mental estão mais associadas aos sintomas do que à severidade do dano labral ou à magnitude das alterações morfológicas.

Sob a perspectiva de uma análise mais complexa, os dados do presente estudo também indicam que, além de variáveis não modificáveis como idade e sexo, a prática de atividade física emergiu como um importante fator correlacionado tanto com menores níveis de dor quanto com maiores níveis de funcionalidade na análise pré-operatória. Partindo do princípio de que pontuações maiores em questionários funcionais são preditoras de bom prognóstico pós-operatório, ${ }^{29}$ e que a prática de atividade física regular é um fator protetor contra dor recorrente após a artroscopia para SIFA, ${ }^{30}$ parece plausível que o engajamento em hábitos de vida mais ativos seja uma recomendação importante por parte dos profissionais que tratam indivíduos com essa condição clínica.

Este estudo apresenta limitações, sendo uma delas o efeito teto apresentado pelo questionário de incapacidade escolhido (HHSm), apesar de haver na literatura ${ }^{9}$ resultados semelhantes também com outros intrumentos, como o Hip Disability and Osteoarthritis Outcome Score (HOOS). De qualquer forma, sugerimos que os resultados deste estudo sejam extrapolados com cautela em casos de outros métodos de quantificação da incapacidade funcional. Além disso, os dados em questão foram extraídos retrospectivamente, o que também sugere que eles sejam interpretados com a devida cautela. Os fatores estruturais avaliados não são todos os existentes relacionados à SIFA, e também existem limitações nas suas avaliações, em especial do ângulo alfa, medido somente por meio de radiografias AP; portanto, a conclusão de que não existe correlação estrutural com o grau de 

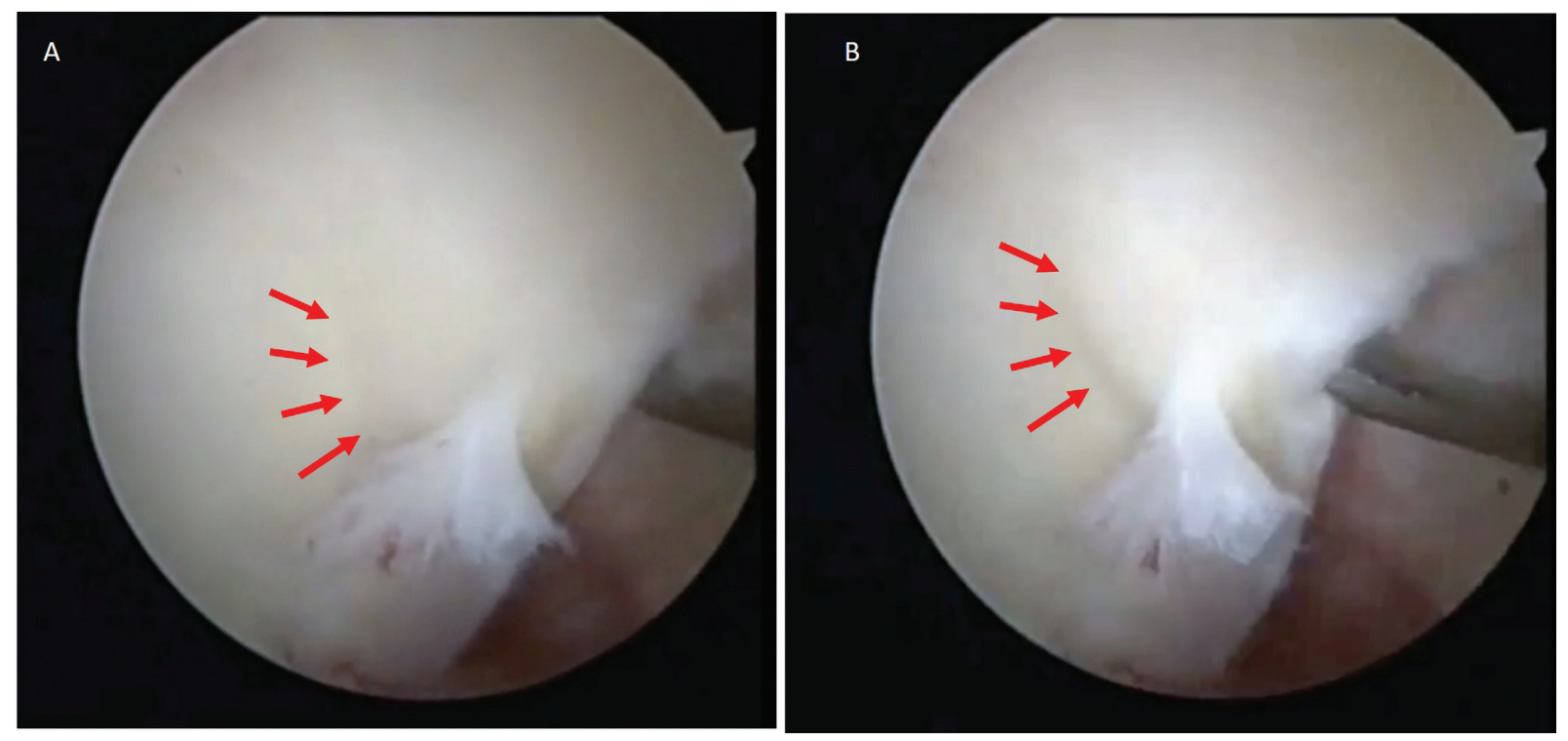

Fig. 1 (A) Setas vermelhas: aspecto da delaminação da cartilagem articular do osso subcondral, característica da lesão em onda. (B) Setas vermelhas: "palpação" da delaminação com sonda artroscópica, confirmando a presença de lesão em onda.

incapacidade deve ser interpretada com base apenas nos métodos utilizados por este estudo.

A falta de avaliação de edema ósseo por ressonância magnética é uma limitação, dado que a literatura descreve que existe maior prevalência deste achado em indivíduos com dor. Mais trabalhos são necessários para avaliar a correlação de outras possíveis variáveis morfológicas, funcionais e multidimensionais com o status funcional e a dor dos pacientes. A SIFA engloba uma gama muito grande de fatores causais, e provavelmente seria necessária a divisão em subgrupos para uma análise mais precisa.

\section{Conclusão}

Não houve correlação do ângulo de Wiberg, do ângulo alfa, e dos danos condrolabrais com a intensidade da dor e o nível de comprometimento funcional nessa coorte de pacientes. $\mathrm{O}$ sexo masculino apresentou menor intensidade da dor, e maiores escores funcionais estão associados ao sexo masculino, menor idade, e à prática de atividade física.

\section{Suporte Financeiro}

Não houve suporte financeiro de fontes públicas, comerciais, ou sem fins lucrativos.

\section{Conflito de Interesses}

Os autores declaram não haver conflito de interesses.

\section{Referências}

1 Ganz R, Parvizi J, Beck M, Leunig M, Nötzli H, Siebenrock KA. Femoroacetabular impingement: a cause for osteoarthritis of the hip. Clin Orthop Relat Res 2003;(417):112-120

2 Freke MD, Kemp J, Svege I, Risberg MA, Semciw A, Crossley KM. Physical impairments in symptomatic femoroacetabular impingement: a systematic review of the evidence [published correc- tion appears in Br J Sports Med 2019;53(20):e7. Br J Sports Med 2016;50(19):1180

3 Kemp J, Grimaldi A, Heerey J, et al. Current trends in sport and exercise hip conditions: Intra-articular and extra-articular hip pain, with detailed focus on femoroacetabular impingement (FAI) syndrome. Best Pract Res Clin Rheumatol 2019;33(01): 66-87

4 Griffin DR, Dickenson EJ, O'Donnell J, et al. The Warwick Agreement on femoroacetabular impingement syndrome (FAI syndrome): an international consensus statement. Br J Sports Med 2016;50(19):1169-1176

5 Beck M, Kalhor M, Leunig M, Ganz R. Hip morphology influences the pattern of damage to the acetabular cartilage: femoroacetabular impingement as a cause of early osteoarthritis of the hip. J Bone Joint Surg Br 2005;87(07):1012-1018

6 Agricola R, Heijboer MP, Bierma-Zeinstra SM, Verhaar JA, Weinans $\mathrm{H}$, Waarsing JH. Cam impingement causes osteoarthritis of the hip: a nationwide prospective cohort study (CHECK). Ann Rheum Dis 2013;72(06):918-923

7 Heerey JJ, Kemp JL, Mosler AB, et al. What is the prevalence of imaging-defined intra-articular hip pathologies in people with and without pain? A systematic review and meta-analysis. Br J Sports Med 2018;52(09):581-593

8 Yamauchi R, Inoue R, Chiba D, et al. Association of clinical and radiographic signs of femoroacetabular impingement in the general population. J Orthop Sci 2017;22(01):94-98

9 Grace T, Samaan MA, Souza RB, Link TM, Majumdar S, Zhang AL. Correlation of Patient Symptoms With Labral and Articular Cartilage Damage in Femoroacetabular Impingement. Orthop J Sports Med 2018;6(06):2325967118778785

10 Clohisy JC, St John LC, Schutz AL. Surgical treatment of femoroacetabular impingement: a systematic review of the literature. Clin Orthop Relat Res 2010;468(02):555-564

11 Yeung M, Kowalczuk M, Simunovic N, Ayeni OR. Hip arthroscopy in the setting of hip dysplasia: A systematic review. Bone Joint Res 2016;5(06):225-231

12 Pimenta CAM, Teixeira MJ. Avaliação da dor. Rev Med (São Paulo) 1997;76:27-35

13 Guimarães R, Alves D, Azuaga T, et al. Translation and transcultural adaptation of the modified Harris Hip Score. Acta Ortop Bras 2009;18(06):339-342 
14 Thompson PD. American College of Sports Medicine. Physical Activity and Fitness Terminology. In: Guidelines for exercise testing and prescription. Baltimore: Wolters Kluwer; 2014:1-18

15 Wiberg G. Studies on dysplastic acetabula and congenital subluxation of the hip joint: with special reference to the complication of osteoarthritis. Acta Chir Scand 1939;83(Suppl):5-135

16 Nötzli HP, Wyss TF, Stoecklin CH, Schmid MR, Treiber K, Hodler J. The contour of the femoral head-neck junction as a predictor for the risk of anterior impingement. J Bone Joint Surg Br 2002;84 (04):556-560

17 Philippon MJ, Stubbs AJ, Schenker ML, Maxwell RB, Ganz R, Leunig M. Arthroscopic management of femoroacetabular impingement: osteoplasty technique and literature review. Am J Sports Med 2007;35(09):1571-1580

18 Barros AAG, Vassalo CC, Costa LP, Gómez-Hoyos J, Paganini VO, Andrade MAP. Reliability of the Arthroscopic Classifications of Hip Chondral Lesions. Rev Bras Ortop (Sao Paulo) 2019;54(04): 440-446

19 Outerbridge RE. The etiology of chondromalacia patellae. J Bone Joint Surg Br 1961;43-B:752-757

20 Konan S, Rayan F, Meermans G, Witt J, Haddad FS. Validation of the classification system for acetabular chondral lesions identified at arthroscopy in patients with femoroacetabular impingement. J Bone Joint Surg Br 2011;93(03):332-336

21 El-Radi MA, Marin-Peña OR, Said HG, Tey-Pons M. Basics in hip chondrolabral lesions and state of the art. SICOT J 2017;3:73

22 Frank JM, Harris JD, Erickson BJ, et al. Prevalence of Femoroacetabular Impingement Imaging Findings in Asymptomatic Volunteers: A Systematic Review. Arthroscopy 2015;31(06):1199-1204
23 KempJL, Collins NJ, Makdissi M, Schache AG, Machotka Z, Crossley K. Hip arthroscopy for intra-articular pathology: a systematic review of outcomes with and without femoral osteoplasty. $\mathrm{Br} \mathrm{J}$ Sports Med 2012;46(09):632-643

24 Minkara AA, Westermann RW, RosneckJ, Lynch TS. Systematic Review and Meta-analysis of Outcomes After Hip Arthroscopy in Femoroacetabular Impingement. Am J Sports Med 2019;47(02):488-500

25 O'Connor M, Minkara AA, Westermann RW, Rosneck J, Lynch TS. Return to Play After Hip Arthroscopy: A Systematic Review and Meta-analysis. Am J Sports Med 2018;46(11):2780-2788

26 Polesello GC, Honda EK, Ono NK, et al. Artroscopia do quadril: experiência após seguimento médio de 33 meses. Rev Bras Ortop 2006;41(05):145-150

27 Potter MQ, Wylie JD, Sun GS, Beckmann JT, Aoki SK. Psychologic distress reduces preoperative self-assessment scores in femoroacetabular impingement patients. Clin Orthop Relat Res 2014;472 (06):1886-1892

28 Jacobs CA, Burnham JM, Jochimsen KN, Molina D IV, Hamilton DA Duncan ST. Preoperative Symptoms in Femoroacetabular Impingement Patients Are More Related to Mental Health Scores Than the Severity of Labral Tear or Magnitude of Bony Deformity. J Arthroplasty 2017;32(12):3603-3606

29 Nabavi A, Olwill CM, Harris IA. Preoperative predictors of outcome in the arthroscopic treatment of femoroacetabular impingement. Hip Int 2015;25(05):402-405

30 Stone AV, Malloy P, Beck EC, et al. Predictors of Persistent Postoperative Pain at Minimum 2 Years After Arthroscopic Treatment of Femoroacetabular Impingement. Am J Sports Med 2019; 47(03):552-559 